\title{
The Chemical Composition of Red Giants, AGB Stars and Planetary Nebulae
}

\author{
Bengt Gustafsson ${ }^{1}$ and Rurik Wahlin ${ }^{1}$ \\ ${ }^{1}$ Department of Astronomy and Space Physics, Uppsala University \\ Box 515, SE-751 20 Uppsala, Sweden \\ email: bg@astro.uu.se
}

\begin{abstract}
The determinations of element abundances in red-giant stars and in particular in AGB stars are reviewed and the resulting abundances are compared with those obtained for planetary nebulae in the Galaxy and in nearby galaxies. The problems, possibilities and implications of such comparisons when estimating yields from low-mass and intermediate-mass stars are illustrated and commented on.
\end{abstract}

Keywords. stars: red giants, abundances, nucleosynthesis; planetary nebulae: abundances; galaxies: evolution

\section{Introduction}

Unlike the theory of stellar evolution, which is often characterized as one of the most successful theories in astronomy, the theory of galaxy evolution is in an unsatisfactory state. The uncertainties as regards star-formation rates and IMF are reflected in free parameters, and also parameters specifying rates of infalling intergalactic gas and mixing of the ISM must be set. When it comes to yields from different types of stars other free parameters must be specified, not the least for the contributions of intermediate- and low-mass stars. Basically, those yields are the result of stellar-evolution calculations but these are, in spite of the general success of that theory, not sufficient or reliable in this respect. Essential uncertainties are stellar mass-loss rates as well as parameters related to the intricacies of mixing processes close to the regions of nuclear processing in the stars. In this situation it would be very important to stabilize the "theory" by some solid observed yields, for SNe, Wolf-Rayet stars, PNe and red giants (Gustafsson 2004). Such observations are, however, also uncertain and dependent on unsatisfactory model atmospheres with additional free parameters.

Attempts to systematically improve this situation, by increasing the accuracy of the model atmospheres and resulting abundance determinations for such objects to infer yields more directly, must be considered to have high priority, not only for learning more about effects of nucleosynthesis in the particular objects but for understanding galaxy evolution as a whole.

In the present review we shall focus on some aspects of such an ambitious programme: the determination of abundances in red giants, including AGB stars, and PNe. Due to space limitations, we shall concentrate on stars with initial masses below approximately $4 \mathrm{M}_{\odot}$, i.e. stars that are not supposed to undergo the Second dredge-up following core $\mathrm{He}$ burning, neither Hot Bottom Burning. This will also exclude comparisons with PNe of Type I, rich in He and N, as well as with PNe with Wolf-Rayet central stars. As regards the recent development in chemical analyses of $\mathrm{PNe}$, we refer to several contributions in the present volume, in particular those of Costa, Dinerstein, Liu, Peimbert, Shaw and Sterling. 
The chemical analysis of red-giant stars presents a number of difficult problems if one wants to reduce systematic errors to a level where also moderate but important effects, e.g. due to internal mixing or dredge-ups, of a factor of two or less in abundance may be traced. Recent observations from major telescopes and spectrometers are of such a high quality in terms of $\mathrm{S} / \mathrm{N}$ that errors in the theoretical analysis, not in the very observations, dominate the error budget for the abundance determinations, at least for stars brighter than $\mathrm{V} \sim 16$. In any analysis, first stellar fundamental parameters such as $\mathrm{T}_{\text {eff }}$, acceleration of gravity at the stellar surface (or mass and radius of the star) must be estimated. For the effective-temperature estimate, interstellar or circumstellar reddening or thermal dust emission in the IR are major systematic sources of error, as well as the effects of uncertainties in the heavy molecular absorption in the stellar photospheres. These may typically lead to errors of $5 \%$ in $\mathrm{T}_{\text {eff }}$ which is significant since the abundance estimates may be very temperature sensitive, not least when derived from the strength of molecular lines. The surface gravity estimate is similarly important - it may often not be obtained accurately from the spectrum but has to be determined from the apparent magnitude and an estimated distance, or just from an adopted absolute magnitude, and an assumed mass. For galactic AGB stars, where the distances may still be quite uncertain as compared with stars in nearby galaxies, the error in the surface gravity may lead to errors in absolute abundances of more than a factor of two, essentially reflecting the pressure sensitivity of molecular equilibria. Still more problematic are the errors in the model atmospheres. The consequences of these problems for chemical analysis have not yet been fully explored. For the Sun, Asplund, Grevesse \& Sauval (2005) have found that proper consideration of the inhomogeneities and the cooling of the upper photosphere due to gas expansion leads to revisions of CNO abundances by about -0.2 dex. The only quantitative study of the effects of adopting such more realistic 3D hydrodynamical models at abundance determinations for red giants is the work by Collet, Asplund \& Trampendach (2006) on the extremely metal-poor giant HE0107-5340 $([\mathrm{Fe} / \mathrm{H}]=-5)$. Collet et al. find that the CNO element abundances, based on diatomic molecules, are reduced by a factor of 10 to 100 when convection is treated more realistically. Effects on abundances based on atomic lines are significantly smaller, typically a factor of two. Although the corresponding effects are probably smaller for metal-rich stars they may be significant. Studies on convection effects on spectra of red supergiants, using the "star in a box" models of (Freytag, in preparation) are presently being pursued. The effects of pulsations on spectra of Mira stars have been explored by Gautschy-Loidl et al. (2004). As regards the model effects of departures from LTE, as well as the uncertainties due to missing or incomplete opacity data and dust formation in the outer stellar layers, they may also be quite considerable (for references, see Gustafsson \& Höfner 2004). Many AGB stars have circumstellar molecular envelopes, and one could wonder whether their $\mathrm{mm}$ and sub-mm lines could offer possibilities to obtain more accurate abundances. A short answer to that question is "No". Abundance estimates from these regions are judged to be accurate to, at best, a factor of 5 or so (Nyman et al. 1993, Woods et al. 2003, Olofsson 2004), and are sometimes in error by one order of magnitude. Isotopic ratios may, however, be accurate to $25 \%$ or even better for some sources, like the famous dust-enshrouded carbon star IRC+10216.

\section{The first dredge-up as traced in observed abundances}

When empirically estimating yields of heavy elements from low-mass and intermediatemass stars, the dredge-ups and mass loss before the AGB stages are important. We shall here briefly summarize the observed evidence for dredge-up episodes before the AGB. 
The first episode, occurring already in the sub-giant phase, is predicted to bring $\mathrm{CN}$ processed material with depleted ${ }^{12} \mathrm{C}$ and correspondingly enhanced ${ }^{14} \mathrm{~N}$, as well as ${ }^{13} \mathrm{C}$, to the stellar surface. The reduction of carbon and enrichment of nitrogen was qualitatively demonstrated for Pop I giants by Lambert \& Ries (1977) and Kjaergaard et al. (1982) but it was found by the latter authors that the nitrogen enrichment was not large enough to match carbon depletion. Recently this mismatch seems to have been unexpectedly resolved by the finding of Asplund et al. (2005) that the solar CNO abundances are significantly lower than earlier believed; this reduces the carbon depletion, and increases the nitrogen enrichment in the red giants, assuming that they initially had solar-like abundances. The ${ }^{13} \mathrm{C}$ enrichment, expected to lead to ${ }^{12} \mathrm{C} /{ }^{13} \mathrm{C}$ ratios of about 20 , was early verified by Dearborn, Lambert \& Tomkin (1975) although some stars were also found with significantly lower ratios, approaching the equilibrium CN-burning value of about 4. Empirically, one also finds stars on the upper giant branch, starting around the bump in the luminosity function, which have depletions in carbon abundances and enhanced nitrogen abundances significantly greater than those predicted (for a recent discussion, see Gratton et al. 2000). Sweigart \& Mengel (1979) earlier suggested that these phenomena might be due to meridional circulation driven by a rapidly spinning stellar core; this may well be the case but, as was recently shown by Palacios et al. (2006), the observed effects seem larger than those expected from evolutionary models with angular-momentum transport and shear instibilities taken into account. Perhaps more detailed models of convection-rotation interaction can produce larger effects.

\section{The third dredge-up: galactic AGB stars vs PNe}

The third dredge-up is generally related to the thermal pulses on the AGB where He is burnt in flashes in a thin shell. The following periodic rise of the temperature gradient above the shell creates a convection zone bringing He-burning products to higher layers from where the outer convection zone may later transport these products to the stellar surface.

Although this general scenario is not strongly disputed, it is as yet not fully understood quantitatively. When Iben (1981) formulated "The Carbon Star Mystery" this was reflecting the fact that the dredge-up of carbon only seemed to occur for core masses of about $0.6 \mathrm{M}_{\odot}$ or more, while the observed luminosity function of carbon stars in the LMC indicated that lower-mass stars may become carbon stars. This problem now seems solved for the LMC, while it still may be hard to obtain the observed low lumnosities of many carbon stars in the SMC (Stancliffe, Izzard \& Tout 2005).

As regards the other characteristic consequences of the third dredge up, the enrichment of the surface layers by s-elements, the situation is theoretically still more unclear. Beautiful empirical studies, observing low $\mathrm{Rb} / \mathrm{Sr}$ ratios and $\mathrm{Mg}$ and $\mathrm{Zr}$ isotopic ratios (Lambert et al. 1995, Smith 2000, Abia et al. 2001), have demonstrated that the s enrichment is mainly the result of the relatively low neutron densities and the ${ }^{13} \mathrm{C}(\alpha, \mathrm{n}){ }^{16} \mathrm{O}$ source. However, the standard models do not produce enough ${ }^{13} \mathrm{C}$ in a hot enough environment. Some extra mixing leading to ingestion of protons into the intershell region would lead to a ${ }^{13} \mathrm{C}$ pocket (Gallino et al. 1998) but also overlaying ${ }^{14} \mathrm{~N}$ which, due to the high cross section of this nucleus for $(n, p)$ reactions, could destroy the neutrons. The blending of rotational mixing and convective overshooting in the bottom of the envelope convection zone with diffuse mixing is not self-consistently handled yet, and the resulting s-element yields are dependent on free parameters, as well as on the angular momentum of the star (cf. Herwig, Langer \& Lugaro 2003, Goriely \& Siess 2004, as well as the contribution by Busso to the present volume). 
In this situation, where the resulting yields are dependent on uncertain, or possibly not even physically fully adequate, parameters in the stellar models, it is particularly important to obtain abundances empirically. For the CNO elements and corresponding isotopes the most complete and accurate studies are still those by Lambert, Smith and collaborators (e.g., Smith \& Lambert 1985, Smith \& Lambert 1986, Lambert et al. 1986, Smith \& Lambert 1990), partially built on analyses of high-resolution FTS spectra in the IR. These authors also compared the CNO abundances of the stars with corresponding data for PNe (see in particular Smith \& Lambert 1990). In addition to a generally good agreement in the abundance patterns, there are some interesting deviations from what might be expected, in particular as regards the results for the N-type carbon stars (Lambert et al. 1986): (1) The nitrogen abundances of these stars are generally lower than those of the M and S stars; in fact N/O is approximately solar, which would not be expected since the stars have gone through the first dredge-up. This N/O distribution, shifted towards low $\mathrm{N}$ abundances, is not reproduced by the carbon-rich PNe, even though there are some nebulae that have lower N/O ratios than the M, MS and S stars tend to show. A systematic uncertainty in the $\mathrm{N}$ abundances of Lambert et al. (1996) is due to the dissociation energy of the $\mathrm{CN}$ molecule (providing the most important $\mathrm{N}$-abundance criteria) but recent new estimates of this quantity do not suggest an important revision downwards which would be needed to reach consistency with the PNe (cf Reddy et al. 2003). A more probable explanation may be errors in the model atmospheres. (2) The distribution of $\mathrm{C} / \mathrm{O}$ ratios of the carbon stars shows a rather narrow peak at ratios between 1.0 and 1.4, while carbon-rich planetary nebulae show a much more extended distribution towards high $\mathrm{C} / \mathrm{O}$ values, even beyond 2 . This might reflect selection effects in the sample of Lambert et al. (1996; the study is limited to the apparently brightest objects in the $\mathrm{K}$ band, and dust enshrouded and presumably quite carbon-rich objects are thus missed). We note that Ohnaka \& Tsuji (1998b) have made an independent analysis of 3 of the stars analysed by Lambert et al. (1996) and obtain significantly higher C/O ratios, which seems to reflect differences in model atmospheres and temperature scales.

Lambert et al. (1996) also provided ${ }^{12} \mathrm{C} /{ }^{13} \mathrm{C}$ ratios, based on several different sets of photospheric molecular IR lines which were found to give mutually consistent results. The results were found to be consistent with an increased $\mathrm{C}$ abundance due to a straight enrichment of ${ }^{12} \mathrm{C}$, with no ${ }^{13} \mathrm{C}$ added in the third dredge-up, excluding the small minority of ${ }^{13}$ C-rich carbon stars ("J-type stars"). Systematically smaller isotopic ratios were obtained for the same stars by Ohnaka \& Tsuji (1996), based on lines in more blended near-IR spectral regions. The methods used by the latter authors were, however, criticised by de Laverny \& Gustafsson (1998); see also Ohnaka \& Tsuji 1998a and de Laverny \& Gustafsson 1999.

Schöier \& Olofsson (2000) and Woods et al. (2003) have derived ${ }^{12} \mathrm{C} /{ }^{13} \mathrm{C}$ ratios for envelopes surrounding galactic carbon stars from mm CO lines. For a number of stars in common with Lambert et al. (1986) the agreement is good. As is seen in Fig. 1a, however, the distribution is different with a larger fraction of stars with lower isotopic ratios. If the stars with high and low mass-loss $(\dot{\mathrm{M}})$ are plotted separately there is also an indication of a steeper slope in the $10 \lesssim{ }^{12} \mathrm{C} /{ }^{13} \mathrm{C} \lesssim 40$ range for the high $\dot{\mathrm{M}}$ sample. The result seems to suggest that the Schöier \& Olofsson (2000) sample is more biased towards low ratios, or possibly that there is a selective depletion of ${ }^{12} \mathrm{C}$ relative to ${ }^{13} \mathrm{C}$ in the envelopes.

${ }^{12} \mathrm{C} /{ }^{13} \mathrm{C}$ ratios have recently been derived for $\mathrm{PNe}$ by several authors, primarily from mm lines (Woods et al. 2005, Balser, McMullin \& Wilson 2002, Josselin \& Bachiller 2003, Bachiller et al. 1997, Palla et al. 2000, Likkel et al. 1988), but also from the hyperfine structure C III] line at $1908 \AA$ (Clegg et al. 1997). The cumulative distribution is shown 

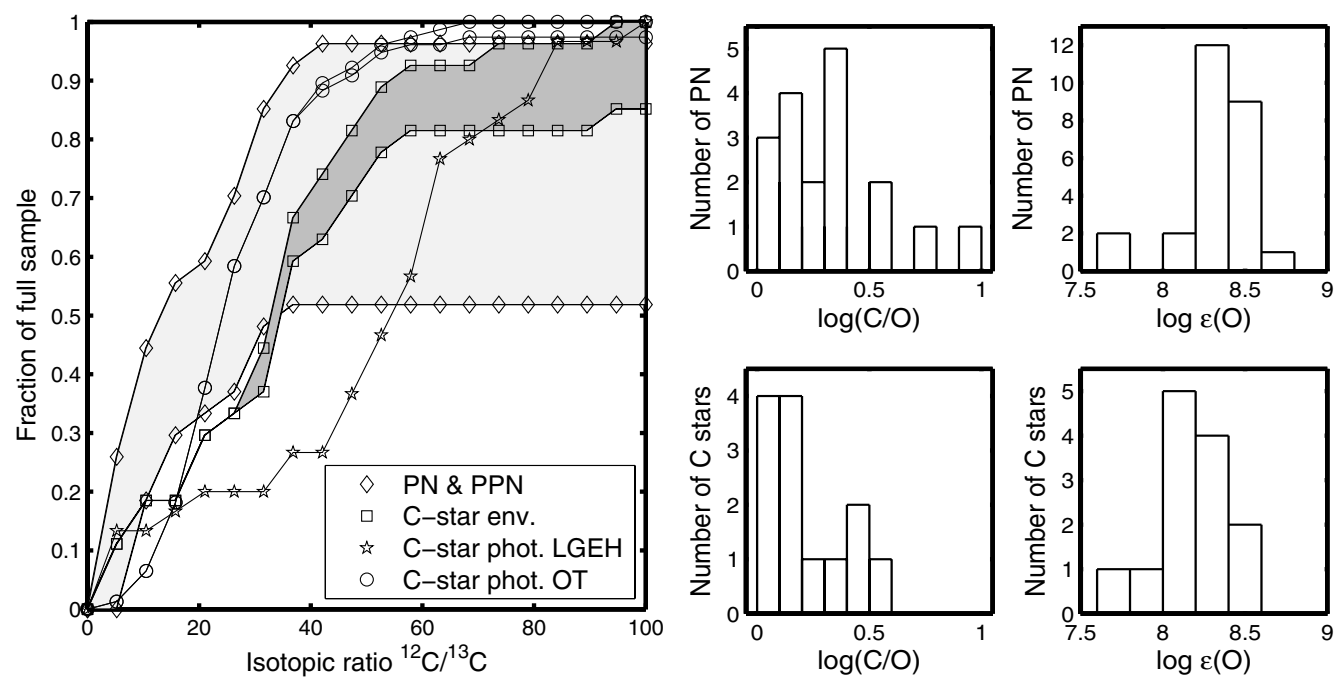

Figure 1. (a; left): The cumulative distribution of ${ }^{12} \mathrm{C} /{ }^{13} \mathrm{C}$ is plotted for different samples. The samples containing lower limit observations give a range of possible values. In the $\mathrm{PNe}$ and PPNe sample only objects with C/O > 1 are included. LGEH represents Lambert et al. (1986), OT Ohnaka \& Tsuji (1996). (b: right): Histograms over C/O ratio and oxygen abundance for carbon rich PN (Stanghellini et al. 2005) and carbon stars (Wahlin et al. 2006) in the LMC. The oxygen abundances are in fair agreement with each other and the $\mathrm{C} / \mathrm{O}$ ratio is on average higher in the PN as expected.

in Fig. 1a for carbon-rich PNe, as analysed by Amnuel, Guseinov \& Rustamov (1989), Lenzuni, Natta \& Panagia (1989), Maciel \& Chiappini (1994), Hasegawa \& Kwok (2003), Costa, Uchida \& Maciel (2004), Cohen \& Barlow (2005) and references therein, when abundances are not given in the same paper as the ${ }^{12} \mathrm{C} /{ }^{13} \mathrm{C}$. This distribution does, however, not depart significantly from the distribution for all PNe with data available. It is seen that the distribution for PNe again contains a greater fraction of stars with relatively low isotopic ratios $\left(10 \lesssim{ }^{12} \mathrm{C} /{ }^{13} \mathrm{C} \lesssim 40\right)$ than the distribution of photospheric ratios. This might be due to selection effects or selective depletion - another possibility is systematic errors in the analysis of the mm line data. We note, however, that the values of Ohnaka \& Tsuji (1996) fit the PNe distribution rather well.

There are several possible explanations for the discrepancy between PNe and typical carbon stars. There could be systematic errors in the observations or analysis giving too low ratios in $\mathrm{PNe}$ and high $\dot{\mathrm{M}}$ carbon shells or too high ratios in the low $\dot{\mathrm{M}}$ carbon stars. Alternatively, the abundances might change when normal carbon stars evolve to high $\dot{\mathrm{M}}$ carbon stars to PNe. This could either be a nuclear evolution producing ${ }^{13} \mathrm{C}$ or possibly destroying ${ }^{12} \mathrm{C}$ or an error in the common assumption that processes dissociating or regenerating ${ }^{12} \mathrm{CO}$ or ${ }^{13} \mathrm{CO}$ in the circumstellar medium balance equally for the two molecules (c.f. Likkel et al. 1988). Another explanation could be that carbon stars such as those observed by Lambert et al. (1986) would often not be progenitors to typical C-rich PNe but instead end without a final intensive wind, and if they produce PNe at all maybe mainly produce spherical ones (cf. Soker 2002). This would then also explain the different morphologies of spherically symmetric shells observed around some bright carbon stars (cf. Olofsson 2004 and references therein) as compared with the normal nonspherical PNe. It should be noted that the distribution relative to galactic longitude of 
the different samples is not different enough to allow the conclusion that the discrepancy could result from the radial gradient of ${ }^{12} \mathrm{C} /{ }^{13} \mathrm{C}$ in the Galaxy (Milam et al. 2005).

Turning to the s-element abundances in PNe Dinerstein (2001) has identified finestructure transitions of Kr III and Se IV in the near IR spectra of planetary nebulae and Sterling, Dinerstein \& Bowers (2002) traced Ge III far-UV lines in several PNe, and possibly even Ga III (Sterling \& Dinerstein 2003). These elements are supposed to be produced early in the s-process. Enrichments of them with the same range as for s-elements in AGB stars are found. Sterling and Dinerstein suggest a variation of the abundances with $\mathrm{C} / \mathrm{O}$ for Se and possibly for $\mathrm{Kr}$ (see present proceedings, their Figure 1). When plotting the corresponding diagrams of the s-element abundances for AGB stars, using the data of Smith \& Lambert (1985), Smith \& Lambert (1986) and Abia et al. (2002), we find a slope for $\mathrm{C} / \mathrm{O}<1.0$, much steeper than the line suggested by Sterling and Dinerstein (but in fact consistent with their data), but no clear increase in $[\mathrm{s} / \mathrm{Fe}]$ for $\mathrm{C} / \mathrm{O}>1.0$. Further work along these lines seems worthwhile.

\section{Local group AGB stars and PNe}

Important further constraints on the third dredge up, as well as on the yields from intermediate- and low-mass stars in the early evolution of galaxies, could be obtained from abundance analyses of AGB stars in Local Group galaxies. Here, the effects of differences in initial abundances may be explored. One example of what may be done is the recent study by de Laverny et al. (2006) of one carbon star in the SMC and two in the Sagittarius dwarf spheroidal, representing stellar populations of different metallicity. The authors obtain abundances of various s elements from high-resolution VLT/UVES spectra, and find that the ratio of high-mass s/low-mass s abundances (e.g. Ba, La, Ce and $\mathrm{Pr}$, relative to $\mathrm{Sr}, \mathrm{Y}$ and $\mathrm{Zr}$ ) seems to vary with metallicity in fair agreement with the variation predicted by Gallino et al. (1998) with a constant value assumed for the mass of the ${ }^{13} \mathrm{C}$ pocket.

In a study of about 50 carbon stars in several local-group dwarf galaxies (Wahlin et al. 2006) we observed spectra in the 1.6 and $2.2 \mu \mathrm{m}$ bands using the VLT/ISAAC and the Phoenix IR spectrometer on Gemini South. We found the oxygen abundance of the program stars to be enhanced on average, compared to the overall metallicity of the host galaxy, by $[\mathrm{O} / \mathrm{Fe}] \sim 0.25$. This is in fair agreement with the overabundance of oxygen as measured in the ISM of such systems (Mateo 1998, Westerlund 1990, Dufour 1984). The $\mathrm{C} / \mathrm{O}$ ratio (by number) increases with decreasing oxygen abundance and with increasing luminosity as

$$
\log (\mathrm{C} / \mathrm{O})=-0.6 \cdot \log \epsilon(\mathrm{O})-0.3 \cdot \mathrm{M}_{\mathrm{bol}}+3.5,
$$

if the few ${ }^{13} \mathrm{C}$ rich stars are excluded. The $\epsilon(\mathrm{O})$ dependence is roughly consistent with the yield estimates of Gavilán, Buell \& Mollá (2005).

The $\mathrm{C} / \mathrm{O}$ ratios of the carbon stars in the LMC are on average higher than the ratios in the galactic carbon stars studied by Lambert et al. 1986, but they are lower than the average C/O of LMC PNe as explored by Stanghellini, Shaw \& Gilmore (2005) (see Fig. 1b). Our preliminary estimates of $\mathrm{C} / \mathrm{O}$ ratios of carbon stars in the Sagittarius dSph are also lower than the $\mathrm{C} / \mathrm{O}$ ratios of its $\mathrm{PNe}$ (Zijlstra et al. 2006) and this also seems to be the case for the Fornax galaxy. The ${ }^{12} \mathrm{C} /{ }^{13} \mathrm{C}$ ratios were found to be high in most carbon stars in the dwarf galaxies, as in their galactic correspondents. The three carbon stars, in the Sagittarius dSph and SMC, studied by de Laverny et al. $(2006)$ show ${ }^{12} \mathrm{C} /{ }^{13} \mathrm{C}>20$, consistent with this picture.

Most stars with relatively high abundance of ${ }^{13} \mathrm{C}$ were found in two distinct areas in the HR diagram. Their $\mathrm{M}_{\text {bol }}$ and $\mathrm{T}_{\text {eff }}$ are consistent with (1) massive evolved stars that could 
have been affected by hot-bottom burning and (2) stars seemingly below the thermally pulsing AGB stars that underwent extrinsic carbon enrichment at low luminosity or were possibly affected by a dredge-up at the He core flash.

Only three of our stars have reliable estimates of the nitrogen abundance, although the uncertainty is probably about 0.5 dex. Two of these have overabundances of nitrogen by $[\mathrm{N} / \mathrm{Fe}] \sim 0.5$. One of them is also enhanced in oxygen which may indicate an Fe abundance higher than assumed on the basis of the overall metallicity of the galaxy. The third star has an overabundance of $[\mathrm{N} / \mathrm{Fe}] \sim 1$. This is probably high enough to be significant and the ${ }^{12} \mathrm{C} /{ }^{13} \mathrm{C} \sim 4$ indicates that the gas in the star has been $\mathrm{CN}$-processed.

\section{Conclusions and prospects}

It should be clear from the discussion above that contemporary results of abundance determinations for AGB stars and for PNe permit interesting comparisons, though still not many firm conclusions. It seems that oxygen abundances of carbon stars and carbon-rich PNe have not been very much affected by intrinsic nucleosynthesis but sooner measure the initial abundances. Also, the shift of the carbon-abundance distribution for carbonrich $\mathrm{PNe}$ relative to the carbon stars indicates that the carbon enrichment proceeds and in many cases reaches its highest value in the superwind, ending the AGB phase and initiating the $\mathrm{PN}$ phase. This is supported also by the observed increase of the $\mathrm{C} / \mathrm{O}$ ratio as a function of carbon-star luminosity. The observed tendency for $\mathrm{C} / \mathrm{O}$ in carbon stars to increase with decreasing initial metallicity is also expected. There are still unsolved problems as regards the unexpectedly low $\mathrm{N}$ abundances obtained for the galactic carbon stars, as well as their high ${ }^{12} \mathrm{C} /{ }^{13} \mathrm{C}$ ratios when compared with $\mathrm{PNe}$; these discrepancies might, however, be due to systematic errors in the analyses. The analysis of AGB star abundances have given very important clues for the understanding of the s process; there is also a general overall consistency between these abundances in PNe and AGB stars, but more data are needed.

In addition to methodological improvements in abundance analysis for stars and $\mathrm{PNe}$, efforts to measure abundances of new elements are important; one obvious example is sulphur in carbon stars, e.g. using CS and CO lines to study the S/O ratio. Also, the possibilities to systematically exploit new wavelength regions, not the least the near and intermediate IR both for stars and PNe, are presently developing and still very promising.

\section{Acknowledgements}

Kjell Eriksson, Susanne Höfner and Nils Ryde are thanked for valuable comments on the manuscript. Albert Zijlstra is thanked for providing data on PNe abundances in the Sagittarius and Fornax systems before publication.

\section{References}

Abia, C., Busso, M., Gallino, R. et al. 2001, ApJ 559, 1117

Abia, C., Domínguez, I, Gallio, R. et al. 2002, ApJ 579, 817

Amnuel P. R., Guseinov O. K., \& Rustamov I. S. 1989, Ap\&SS 154, 21

Asplund, M., Grevesse, N., \& Sauval, A.J. 2005, ASPC 336, 25

Bachiller, R., Forveille, T., Huggins, P.J., \& Cox, P. 1997, A $\& A$ 324, 1123

Balser, D.S., McMullin, J.P., \& Wilson, T.L. 2002, ApJ 572, 326

Clegg, R.E.S., Storey, P.J., Walsh, J.R., \& Neale L. 1997, MNRAS 284, 348

Cohen, M. \& Barlow, M.J. 2005, MNRAS 362, 1199

Collet, R. Asplund, M., \& Trampendach, R. 2006, ApJ, 644, L121

Costa, R.D.D., Uchida, M.M.M., \& Maciel W. J. 2004, A\& A 423, 199 
Dearborn, D.S.P., Lambert, D.L., \& Tomkin, J. 1975, ApJ 200, 675

de Laverny, P. \& Gustafsson, B. 1998, A\&A 332, 661

de Laverny, P. \& Gustafsson, B. 1999, A\&A 346, 520

de Laverny, P., Abia, C., Domínguez, I. et al. 2006, A\& A 446, 1107

Dinerstein, H.L. 2006, ApJ 550, L223

Dufour, R.J. 1984, IAUS 108, 353

Gallino, R., Arlandini, C., Busso, M., et al. 1998, ApJ 497, 388

Gautschy-Loidl, R., Höfner, S., Jørgensen, U.G., \& Hron 2004, A\&A 422, 289

Gavilán, M., Buell, J.F., \& Mollá, M. 2005, A\&\&A 432, 861

Goriely, S. \& Siess, L. 2004, A\&A 421, L25

Gratton, R.G., Sneden, C., Carretta, E., \& Bragaglia, A. 2000, A $\& A$ 354, 169

Gustafsson, B. 2004, in The Future Astronuclear Physics, eds. A. Jorissen et al., EGAS Publications Series 11, 21

Gustafsson, B. \& Höfner, S. 2004, in Asymptotic Giant Branch Stars, eds. H.J. Habing, H. Olofsson, E. Griffin, Springer, p. 149

Hasegawa, T. I. \& Kwok, S. 2003, ApJ 585, 475

Herwig, F., Langer, N., \& Lugaro, M. 2003, ApJ 593, 1056

Iben, I., Jr. 1981, ApJ 246, 278

Josselin, E. \& Bachiller, R. 2003, A\&A 397, 659

Kjaergaard, P., Gustafsson, B., Walker, G.A.H., \& Hultqvist, L. 1982, A\& A 115, 145

Lambert, D.L. \& Ries, L.M. 1977, ApJ 217, 508

Lambert, D.L., Gustafsson, B., Eriksson, K., \& Hinkle, K.H. 1986, ApJS 62, 373

Lambert, D.L, Smith, V.V., Busso, M., et al. 1995, ApJ 450, 302

Lenzuni, P., Natta, A., \& Panagia N. 1989, ApJ 345, 306

Likkel, L., Morris, M., Forveille, T., \& Omont A. 1988, A $E A$ A 198, L1

Maciel, W. J. \& Chiappini, C. 1994, Ap\&SS 219, 231

Mateo, M. 1998, ARA $\& A$ 36, 435

Milam, S. N., Savage, C., Brewster, M. A., \& Ziurys, L. M. 2005, ApJ 634, 1126

Nyman, L.-A, Olofsson, H., Johansson, L.E.B., et al. 1993, A\&\&A 269, 377

Ohnaka, K. \& Tsuji, T. 1996, A\&\&A 310, 933

Ohnaka, K. \& Tsuji, T. 1998a, A\&A 335, 1018

Ohnaka, K. \& Tsuji, T. 1998b, IAU Symp 191 Poster Session P1-16

Olofsson, H. 2004, in Asymptotic Giant Branch Stars, eds. H.J. Habing et al., Springer, p. 325

Palacios, A., Charbonnel, C., Talon, S., \& Siess, L. 2006, A\&A 453, 261

Palla, F., Bachiller, R., Stanghellini, L., Tosi, M., \& Galli, D. 2000, A $\& A$ 355, 69

Reddy, R.R., Nazeer Ahammed, Y., Rama Gopal, K., \& Baba Basha, D. 2003, Ap\&SS 286, 419

Schöier, F.L. \& Olofsson, H. 2000, A\&A 359, 586

Smith, V.V. \& Lambert, D.L. 1985, ApJ 294, 326

Smith, V.V. \& Lambert, D.L. 1986, ApJ 311, 843

Smith, V.V. \& Lambert, D.L. 1990, ApJS 72, 387

Smith, V.V. 2000, IAU Symp 17r, 443

Soker, N. 2002, A $\& A 386,885$

Stancliffe, R.J., Izzard, R.G., \& Tout, A. 2005, MNRAS 356, L1

Stanghellini, L., Shaw, R.A., \& Gilmore, D. 2005, ApJ 622, 294

Sterling, N.C., Dinerstein, H.L., \& Bowers, C.W. 2002, ApJ 578, L55

Sterling, N.C. \& Dinerstein, H.L. 2003, RMxAC 18, 133

Sweigart, A.V. \& Mengel, J.G. 1979, ApJ 229, 642

Wahlin, R., Eriksson, K., Gustafsson, B. et al. 2006, Proc. VIII Torino Workshop on Nucleosynthesis in AGB Stars, Mem Soc Astr. Ital., 77, 955

Westerlund, B. 1990, A\&ARv 2, 29

Woods P. M., Nyman L.-Å., Schöier F. L., et al. 2005, A\&گA 429, 977

Woods, P.M., Schöier, F.L., Nyman, L.-A., \& Olofsson, H. 2003, A\&\}A 402, 617

Zijlstra, A.A., Gesicki, K., Walsh, J.R. et al. 2006, MNRAS 369, 875 